\title{
Nicotine replacement therapies: smoking cessation outcomes in a pharmacy setting in Scotland
}

\author{
Hazel K Sinclair, Christine M Bond, A Scott Lennox, Ross J Taylor, Arthur J Winfield
}

\begin{abstract}
Objective - To determine the volume of sales in Scotland of the various types of nicotine replacement therapy (NRT), the user population demography, and smoking cessation outcome.
\end{abstract}

Methods - A multistage design was used. A questionnaire was sent to a $50 \%$ random sample of the 1139 registered Scottish community pharmacies to monitor NRT availability and to recruit to the second stage. A second pharmacy stage assessed volume and nature of sales and collected customer information. Customers were followed up by postal questionnaire at one month, and by telephone interview or postal questionnaire at seven months.

Results - Of the pharmacies initially contacted, $80 \%$ completed the first questionnaire and $52 \%$ completed the records of NRT sales. The initial point-of-sale questionnaire was completed by $42 \%$ of the customers, $22 \%$ responded at one month, and $21 \%$ at seven months. On average, pharmacies sold five NRTs per week, with patches accounting for $52 \%$ of sales, gum $37 \%$, and others $11 \%$. The mean age of customer respondents was 43 years; most were female (59\%), affluent $(53 \%)$, regularly used the same pharmacy $(88 \%)$, and had received pharmacy support $(72 \%)$. Subjects lost to follow up were assumed to have continued to smoke. The point prevalence cessation rate at one month was $15 \%(13 \%$ excluding long term gum users), and $9 \%$ (6\% excluding long term gum users) achieved seven months of continuous abstinence. Most telephone interviewees $(90 \%)$ would value a more structured pharmacy counselling service.

Conclusions - The methodological difficulties of pharmacy based research are highlighted, in particular the problem of following up customers in the pharmacy setting. However, in spite of the poor customer response, the results have face validity in that they concur with previously published data on NRT use in other settings. Smoking cessation rates for customers buying NRT in community pharmacies seem to be comparable to those previously reported for other set- tings. There is a demand from NRT users for the introduction of a more structured pharmacy counselling service, which now needs to be developed and evaluated.

(Tobacco Control 1995; 4: 338-343)

Keywords: smoking cessation, community pharmacy, nicotine replacement therapy

\section{Introduction}

Initially, nicotine replacement therapy (NRT) was available only on prescription from doctors. Placebo controlled trials, in the setting of general practice, have already shown that both gum and patches make a significant contribution to smoking cessation..$^{1-3}$

In 1991 nicotine gum became available in Scotland under the supervision of the pharmacist, without a requirement for medical backup, and in the following year patches could also be sold over the counter. Little attention has been given to the potential role of the community pharmacist as a provider of support and counselling on smoking cessation, the majority of published studies having concentrated on the nursing and medical members of the primary health care team.

Health promotion is now a contractual obligation for community pharmacists, and the majority are enthusiastic about taking on this role. ${ }^{4-6}$ However, concerns have been expressed that pharmacists are not trained for smoking cessation counselling and that it may be seen as conflicting with the general practitioner, who may even feel threatened. ${ }^{7.8}$ Another possible disadvantage of the over the counter sale of NRT products is that patients are not attached to a particular pharmacy and are therefore difficult to follow up.

Unlike previous studies of NRT use, this study had a community pharmacy setting and we aimed to assess the effect of the recent deregulation of NRTs in relation to smoking cessation outcome. We monitored the volume of sales in Scotland of the various types of NRT, the user population demography, and information on current and potential pharmaceutical support.

\section{Methods}

This study was carried out in community pharmacies - that is, all pharmacies that de- 
liver pharmaceutical care in a primary health care setting. A multistage design was used : two pharmacy stages and three customer stages. A pilot study carried out in 10 local pharmacies highlighted the problem of poor customer response, with only 23 of $85(27.1 \%)$ completing the questionnaire in the pharmacy.

The first pharmacy stage was a questionnaire sent in September 1993 to a $50 \%(n=570)$ random sample of the 1139 registered community pharmacies in Scotland. The sample was stratified by health board. Pharmacists were asked for information on NRT items stocked and displayed, whether they recorded NRT sales on their patient medication records (PMRs), their provision of antismoking leaflets, and demographic factors (pharmacy type and location). Response rates were maximised by sending two postal reminders. All 570 pharmacies were invited to participate in the second pharmacy stage.

Second pharmacy stage recruits $(n=351)$ were asked to tally all NRT sales during one working week and to request all NRT customers to complete a point-of-sale smoking history questionnaire in the pharmacy. Pharmacies were given a postal reminder and telephone follow up. In an effort to overcome the problem of poor customer response identified during the pilot, pharmacy staff involvement and motivation were encouraged by emphasising the importance of every customer sale and supplying bright green prompt labels for prominent display beside the NRT stocks. The point-of-sale smoking history questionnaire collected information on user demography (gender, age, and postcode as a proxy for socioeconomic status ${ }^{9}$ ), smoking history (duration of smoking and cigarette consumption), and details on stopping smoking (previous attempts, product purchased, start of course or renewal of supply, and product publicity). The questionnaire requested respondents to give their name and contact address if they were willing to complete a follow up postal questionnaire.

Subjects who provided a contact address were sent a one-month follow up postal questionnaire; two postal reminders were given. Information was sought about their experience in the pharmacy at the first purchase (whether questioned about their health; advice on product strength and on how to use the product). Initial confidence in the product was assessed using a five point Likert rating scale (very confident, confident, unsure, fairly sceptical, and very sceptical), and users were asked whether they had read and complied with the manufacturer's product information. The questionnaire also asked if they had received product advice from other health care professionals, duration of product use, and current smoking status (point prevalence). Respondents were asked to give a daytime and/or evening telephone contact number if they were willing to be followed up by telephone interview.

A second follow up was conducted seven months after the initial NRT purchase (four months after completion of the theoretical three month course of NRT), because those refraining from smoking for four months have an $80 \%$ chance of becoming long term nonsmokers. ${ }^{10}$ Those one-month respondents who had provided a contact telephone number were followed up with an in depth, semistructured telephone interview. Interviewees were asked about their current smoking status and duration of continuous abstinence, duration of NRT use, their opinions of the utility of the product, and pharmacy support experienced. They were also asked for their views on two potential methods of pharmaceutical support (more structured, regular individual counselling from pharmacy personnel or a support group led by the pharmacist). The interviews were taped and transcribed. Subjects who were unable or unwilling to be contacted by telephone were followed up by postal questionnaire; to optimise response, those who had not responded at the one-month follow up were also sent this short questionnaire to determine current smoking status and duration of continuous abstinence, duration of NRT use, and their opinions of the usefulness of the treatment; two postal reminders were given.

Throughout the study smoking status was self reported; recent evidence ${ }^{11}$ suggests that biochemical validation is not justified for minimal intervention studies. Subjects lost to follow up were regarded as having returned to smoking.

A standard statistical package ${ }^{12}$ was used for data storage and the computation of descriptive statistics.

Definitions of self report measures were as follows ${ }^{11}$ :

Point prevalence - the percentage of former smokers who are not smoking at a particular point in time.

Continuous abstinence - the percentage of former smokers who have not smoked at all since the time of intervention.

\section{Results}

RESPONSE RATES

The response rates for the two pharmacy stages are shown in table 1 . Of the 569 pharmacies contacted, 455 completed the first pharmacy stage questionnaire; 351 outlets were recruited to the second pharmacy stage, and 298 subsequently completed this stage $(84.9 \%$ of recruits or $52.4 \%$ of the pharmacies initially contacted).

The customer response rates are shown in table 2 . There were 1415 recorded sales during the study week and $599(42.3 \%)$ customers completed the point-of-sale smoking history questionnaire. As the number of sales per pharmacy increased, the customer response rate decreased; for example, the pharmacy with the highest number of sales returned only three questionnaires from 65 sales $(4.6 \%$ response), four questionnaires were completed in an outlet that sold 47 NRTs $(8.5 \%$ response), and none were completed in a pharmacy with 27 sales, while 30 of the 35 pharmacies that sold one NRT returned a completed customer questionnaire. 
Table 1 Pharmacy response rates

\begin{tabular}{lllr}
\hline & \multicolumn{1}{c}{ Action } & Response, $\%(n)$ & Response, $\%$ ॠ \\
\hline 1st Pharmacy Stage & Postal questionnaire & $80.0(455 / 569)$ & 80.0 \\
2nd Pharmacy Stage & Recruited to 2nd stage & $77.1(351 / 455)$ & 61.7 \\
\hline
\end{tabular}

* Denominator: 569 initially contacted pharmacies.

Table 2 Customer response rates

\begin{tabular}{|c|c|c|c|}
\hline & Action & Response, $\%(n)$ & Response, $\%$ * \\
\hline \multirow[t]{2}{*}{$\begin{array}{l}\text { Point-of-sale } \\
\text { smoking history }\end{array}$} & Point-of-sale questionnaire & $42.3(599 / 1415)$ & 42.3 \\
\hline & Contact address given & $67.8(406 / 599)$ & 28.7 \\
\hline \multirow{2}{*}{$\begin{array}{l}\text { First follow up } \\
\text { (one-month) }\end{array}$} & One-month postal questionnaire (268) & & \\
\hline & $\begin{array}{l}\text { One-month outcome data provided } \\
\text { retrospectively at seven months }(47) \\
\text { Contact telephone number given }\end{array}$ & $\begin{array}{l}77.6(315 / 406) \\
71.3(191 / 268)\end{array}$ & $\begin{array}{l}22.3 \\
13.5\end{array}$ \\
\hline $\begin{array}{l}\text { Second follow up } \\
\text { (seven-month) }\end{array}$ & $\begin{array}{l}\text { Seven-month: telephone interview (191) } \\
\text { postal questionnaire (103) }\end{array}$ & $72.4(294 / 406)$ & 20.8 \\
\hline
\end{tabular}

^ Denominator: 1415 recipients of NRT.

A contact address was provided by 406 users. The one-month follow up postal questionnaire was completed by 268 users and 191 gave a follow up telephone contact number. One-month outcome data were gathered for a further 47 subjects who had not responded to the one-month postal questionnaire but subsequently completed the seven-month postal follow up. Seven-month follow up data were collected on 294 users: 191 telephone interviews and 103 postal respondents $(20.8 \%$ of the original 1415 NRT users).

\section{COMMUNITY PHARMACY RESPONDENTS}

The composition of the one-month follow up respondents is described in table 3 . The distribution of pharmacy types and location closely parallels the findings of a previous Scottish study ${ }^{4} ;$ most were single outlets and the majority were located in a town.

USER POPULATION CHARACTERISTICS

The majority of the one-month follow up respondents were female $(354 / 599,59.1 \%)$. Age ranged from 17 to 82 years, with a mean of 43.0 years for males and 43.7 years for females. Respondents were more affluent than the Scottish population as a whole, with $52.7 \%$ of the study sample, compared to $41.6 \%$ of Scotland, having a Carstairs-Morris socioeconomic status score ${ }^{9}$ of less than 4 . They had been smokers for between two and $60+$ years (mean 24 years) and had previously smoked between three and $80+$ cigarettes per day (mean 24$)$. The majority $-475 / 599(80.1 \%)-$ had tried at least once before to stop smoking.

Table 3 Description of one-month follow up respondents

\begin{tabular}{lcll}
\hline Type & $\%_{o}(n=455)$ & \multicolumn{1}{c}{ Location } & $o_{o}(n=455)$ \\
\hline National multiple $^{1}$ & $27.9(127)$ & City/suburban & $32.5(148)$ \\
Small multiple $^{2}$ & $29.9(136)$ & Town $^{3}$ & $50.3(229)$ \\
Single outlet & $42.2(192)$ & Rural & $17.2(78)$ \\
\hline
\end{tabular}

${ }^{1}>9$ branches ${ }^{2} 2-9$ branches ${ }^{3} 4000-90000$ inhabitants.
NRT SALES

A total of 1415 NRT sales was recorded by the 298 pharmacies during the study week; 1300 $(91.9 \%)$ were OTC sales and $115(8.1 \%)$ private prescriptions. The number of sales per pharmacy ranged from 0 to 65 (mean 4.7 ); 84 $(28.2 \%)$ pharmacies had no sales. Patches accounted for $51.7 \%$ of the sales, gum $37.5 \%$, and "other" products $10.8 \%$. The most commonly purchased patches were the 7-day packs of the three strengths of Nicotinell (24 hour) (456 sales), and Nicorette (16 hour) (214 sales).

NRT PRODUCTS

Most respondents - 331/599 (55.3\%)learned of their product through advertising, with television being specified by $213(35.6 \%)$. A quarter $(24.7 \%)$ received information about the product from a health care professional (doctor $14.2 \%$, pharmacist $8.3 \%$, occupational nurse $1.3 \%$, health board $0.9 \%$ ), while $19.2 \%$ learned of it from a relative or friend. Almost all interviewees (174/191, $91.1 \%$ ) would recommend the product they had used, the majority $(162,84.8 \%$ ) having already done so.

Most (543/599, 89.1\%) were buying the product for their own use. The majority $(377 / 599,63.0 \%)$ were renewing their supply of NRT, while $222(37.0 \%)$ were starting a course of treatment.

When they first bought the product, 119 of $268(44.4 \%)$ one-month respondents were very or fairly confident that it would help, $44.0 \%$ were unsure, and $11.6 \%$ were fairly or very sceptical. Subsequently, at the sevenmonth follow up, 254 of $294(86.4 \%)$ thought the NRT had helped, $16(5.4 \%)$ thought it had not helped, and $24(8.2 \%)$ were unsure.

Almost all $(264 / 268,98.5 \%)$ had read the product information; however, only 158 $(59.0 \%)$ followed the manufacturer's recommendations fully, $97(36.2 \%)$ complied partially, and eight $(3.0 \%)$ did not follow the recommendations. In response to a further 
specific question on concurrent smoking, 87 $(32.5 \%)$ had smoked at some time when using the product.

At the seven-month follow up $49.1 \%$ of the gum users $(52 / 106)$ were still using the product to some extent, 12 were using a combination of cigarettes and gum, while no respondents were still using patches. Some interviewees felt they had transferred their nicotine addiction from cigarettes to gum and found their new addiction even more difficult to break; several suggested that a nicotine-free placebo gum may help. Others said they were not addicted to the gum's nicotine but used it to compensate for the "habit" of smoking, chewing the same piece for much of the day.

\section{SMOKING CESSATION OUTCOME}

Subjects lost to follow up $(1100 / 1415[77.7 \%]$ at one month, and $1121 / 1415(79.2 \%)$ at seven months) were assumed to have lapsed. Point prevalence cessation was self reported by $212 / 1415(15.0 \%)$ subjects at one month. Continuous abstinence throughout the previous seven months was reported by 127 $(9.0 \%)$ at the seven-month follow up; however, this included 40 long term gum users who were still using the product to some extent at seven months. Excluding these long term users gives a one-month point prevalence cessation rate of $12.5 \%(172 / 1,375)$ and a continuous abstinence rate of $6.3 \%(87 / 1375)$ at seven months.

Almost all interviewees (96/191, 99.0\%) who had failed at this attempt intended to try again. Thirteen $(13.4 \%$ ) were currently on a new attempt. Most - $53(54.6 \%)$ - intended to use patches, and $12(12.4 \%)$ gum, while 21 $(21.7 \%)$ intended to try without using NRT

MEDICAL AND NURSING SUPPORT

A third $(92 / 268,34.3 \%)$ of the one-month respondents had received product advice from their family doctor, three $(1.1 \%)$ from a practice nurse, and two $(0.7 \%)$ from a health visitor. Family doctors provided 59 respondents $(22.0 \%$ ) with additional suggestions for smoking cessation, seven $(2.6 \%)$ received suggestions from a practice nurse, and four $(1.5 \%)$ from a health visitor. Only three respondents $(1.1 \%$ ) were currently attending an antismoking clinic.

\section{PHARMACY SUPPORT}

Most pharmacies $(442 / 455,97.1 \%)$ stocked antismoking leaflets, $425(93.4 \%)$ displaying this health promotion material in the front shop. The majority $(353,77.6 \%)$ did not record any NRT sales on computer, while 70 $(15.4 \%)$ used their patient medication records (PMRs) to record prescribed NRTs.

When first purchasing the product, 140 of the 268 second customer postal questionnaire respondents $(52.2 \%)$ reported that they had been advised by the pharmacist or pharmacy assistant on product strength, and $102(38.1 \%)$ reported that they had been counselled on how to use the product; the majority $(190,70.9 \%)$

did not recall being asked about their health.

Most telephone interviewees (142/191, $74.3 \%$ ) used the same pharmacy for each NRT purchase, $26(13.6 \%)$ used either of two local outlets, $23(12.1 \%)$ did not have a regular pharmacy.

When asked, "Did the pharmacy personnel seem interested in your attempt to stop smoking?" $97(50.7 \%)$ thought they were always interested, as illustrated by :

"They were always interested. It gave you an incentive to be able to talk about it... The pharmacist was technical, the assistants were more for you ... I found it quite helpful."

A further $28(14.7 \%)$ thought they sometimes showed an interest, and $13(6.8 \%)$ received counselling before their first purchase only. However, 51 users $(26.7 \%$ ) did not recall receiving any counselling, as illustrated by:

"Not really, it was just like selling a loaf of bread or pint of milk, kind of thing."

(Two users never visited a pharmacy, the product being purchased on their behalf.) Almost all $(129 / 138,93.5 \%)$ of those who received any counselling felt it had been helpful.

\section{POTENTIAL METHODS OF PHARMACEUTICAL} SUPPORT

Most interviewees $(171 / 191,89.5 \%)$ thought more structured regular individual counselling from the pharmacist or specially trained pharmacy staff would be beneficial, $13(6.8 \%)$ felt it would not help them, while seven $(3.7 \%$ ) were unsure. Several in favour of pharmacy counselling highlighted the lack of privacy in traditional pharmacies. The majority (124, $64.9 \%$ ) would have been interested in attending a support group led by the pharmacist. Most $(111,58.1 \%)$ felt the main benefit would be long term mutual support. Eighty five $(44.5 \%)$ favoured regular individual counselling, $45(23.6 \%)$ a support group, 51 $(26.7 \%)$ liked both equally, while $10(5.2 \%)$ felt neither would be beneficial.

\section{Discussion}

Most previous studies of the effectiveness of NRT have been carried out on populations that are not representative of typical smokers. Many used subjects at smoking clinics, who are likely to be highly motivated, ${ }^{1-3}$ and even the few studies of cessation unaided by health professionals drew their subjects from volunteers. ${ }^{13,14}$ Our study aimed to capture a population representative of smokers who attempt to stop smoking on their own with the aid of NRT.

However, our study encountered major difficulties in recruiting a representative sample of pharmacy NRT purchasers. There could be several reasons for poor customer recruitment. Firstly, the doctor-patient relationship differs from the pharmacist and his customer $^{15,16}$ such that the client may feel 
more at liberty to refuse the pharmacist's invitation to complete a questionnaire. Secondly, unlike the medical setting, the pharmacy environment frequently lacks privacy. ${ }^{17}$ Thirdly, the poor response rate may be largely due to the pharmacist rather than the customer. The pharmacies with the highest number of sales returned relatively few completed questionnaires. A recent study in Minnesota (USA) showed that $43 \%$ of pharmacists said they lacked the time required to give advice, while one in five rated giving smoking cessation advice as a low priority for them ${ }^{6}$; this may account for the particularly poor response rates in the busiest outlets, where customers may not have been offered the questionnaire. Moreover, unlike general practices, pharmacies do not have exclusively registered populations; customers can choose to avoid a pharmacy where they are asked to complete questionnaires.

The problem of poor response was exacerbated by the further attrition of the sample at the one-month and seven-month follow up, achieving overall response rates of only $22 \%$ and $21 \%$, respectively (see table 2 ).

Our strategy to improve the customer response rate in the pharmacy was to encourage pharmacy staff involvement and motivation by emphasising the importance of every customer sale, and to supply prompt labels for prominent display beside the NRT stocks. Other potential strategies (for example, prize draws) may be needed to increase both staff and customer motivation and so improve response rates.

The results can therefore be considered in two sections: first, those based on the initial survey, which achieved an acceptable response and can therefore be said to represent the population of Scottish pharmacists; second, those based on the sample of aspiring nonsmokers, partially self selected but also possibly selected opportunistically by the pharmacy personnel. Despite the indeterminate biases resulting from client self selection and pharmacist customer selection, and the attrition at the one-month and seven-month follow up, we still consider that the descriptive data and self reported abstinence are worth reporting, given the dearth of similar research data. ${ }^{18-20}$ This paper also highlights the inherent difficulty of applying scientific rigour to the assessment of therapeutic efficacy in a commercial retail versus a medical setting.

Since the Scottish deregulation of $2 \mathrm{mg}$ Nicorette gum in May 1991 and nicotine containing patches in November 1992, the supervision of NRT sales has moved rapidly from general practitioners to pharmacists. From October to December 1993 there were 5000 NRT sales each week and the estimated period prevalence of NRT use by smokers was $0.5 \%$, given 1139 community pharmacies and approximately one million smokers in Scotland. With $60 \%$ of the population visiting a community pharmacy at least once a month, ${ }^{21}$ pharmacists are ideally placed for opportunistic health promotion; indeed, health promotion is now a contractual requirement. In recognition of this role, almost all the pharm- acies (97\%) stock antismoking leaflets, 93\% displaying them in the front shop.

As more drugs are deregulated and the supervision of more medicines moves from the GP to the pharmacist, the professionally stimulating ${ }^{4-6}$ but time consuming role for the community pharmacist in overseeing all pharmacy supervised sales becomes ever more difficult to implement. In recognition, the Royal Pharmaceutical Society has recently circulated guidelines on smoking cessation advice in the pharmacy. ${ }^{22}$ However, most of the pharmacist's income is derived from dispensing fees and over the counter sales, and advice is given to the public free of change. In contrast, the remuneration structure for community pharmacists in Quebec has been revised to provide payments for professional advice. ${ }^{23}$ A similar policy change in Britain might reduce the commercial conflict factor and so facilitate the expansion of the pharmacist's role in health promotion; for example, pharmacy personnel might be more likely to advise a customer who was not really motivated to stop smoking of the inappropriateness of making an NRT purchase. The profession, as represented by its societies, is in favour of extending the advisory role and the introduction of a more realistic remuneration package. ${ }^{24}$

Although almost all respondents read the product information, only $59 \%$ complied fully with the manufacturer's recommendations, a third having smoked at some time when using the product. The problem of poor customer compliance has been highlighted in previous studies. ${ }^{18,19}$ Because smoking during the second week of treatment has been shown to be a powerful predictor of failure, ${ }^{18}$ pharmacy personnel should stress the importance of total abstinence to a successful smoking cessation effort. They could also use the pharmacy computer to record the use of NRTs, so enabling pharmacy personnel to follow the user's progress and provide continuing counselling, which could result in more successful outcomes.

A recent review suggested that $4 \mathrm{mg}$ gum was the most effective form of replacement for the most highly dependent smokers (those craving a cigarette on waking), while in less dependent smokers the different preparations were comparable in efficacy. ${ }^{2}$ However, there is a risk that some users may transfer their nicotine addiction to the gum $^{20}$; our study found NRT gum, but not patches, appeared to be associated with long term use. The introduction of a lower strength or nicotine-free (placebo) gum may help those who feel they have transferred their nicotine addiction from cigarettes to gum.

Even allowing for the confounding factor of lack of sample representativeness discussed in detail earlier, this study has shown that the pharmacy supervised sale of NRTs is making a significant contribution to achieving the government's targets on reducing smoking prevalence, ${ }^{25}$ achieving a point prevalence smoking cessation rate at one month of $15 \%$ (13\% excluding long term gum users) and a 
continuous abstinence rate of $9 \%(6 \%$ excluding long term gum users) at seven months.

Three quarters of subjects had received some pharmacy counselling, and of these, $93 \%$ found this helpful. However, there is wide interest among NRT users in more structured pharmacy counselling. In conjunction with local health promotion advisers we have launched a randomised controlled trial to evaluate such a service, emphasising the importance of motivation, ${ }^{26,27}$ monitoring compliance, and providing encouragement; this is ongoing. Such a minimal intervention applied nationally could maximise the potential of NRT and would be expected to be highly costeffective.

We are grateful to the Scottish Office, Home and Health Department for funding this project; however, the views expressed in this article are solely those of the authors. We are also grateful to the large number of pharmacists, pharmacy staff, and NRT users who made this study possible by responding to our requests for information. No pharmaceutical company support was received.

1 Imperial Cancer Research Fund General Practice Research Group. Effectiveness of a nicotine patch in helping people stop smoking: results of a randomised trial in genera practice. $B M \mathcal{F}$ 1993; 306: 1304-8.

2 Tang JL, Law $M$, Wald $N$. How effective is nicotine replacement therapy in helping people to stop smoking ? $B M \mathcal{F} 1994$; 308: 21-6.

3 Nicorette: an update. Drug Ther Bull 1985; 23 : 67-8.

4 Bond CM, Sinclair HK, Winfield AJ, Taylor RJ. Community pharmacists' attitudes to their advice giving role munity pharmacists' attitudes to their advice giving role and the deregulat 2 : $26-30$.

5 Report by the Controller and Auditor General. Community pharmacies in England. London: National Audit Office, 28 May 1992

6 Martinez PJ, Knapp J, Kottke TE. Beliefs and attitudes of Minnesota pharmacists regarding tobacco sales and smoking cessation counselling. Tobacco Control 1993; 2 : 306-10.

7 Roberts D. Dispensing by the community pharmacists: an unstoppable decline? $\mathcal{F} R$ Coll Gen Pract 1988; 38:563-4.

8 Eaton G, Webb B. Boundary encroachment: pharmacists in the clinical setting. Sociol Health Illness 1979; 1: 69-89.

9 Carstairs V, Morris R. Deprivation and health in Scotland. Aberdeen: Aberdeen University Press, 1991; 305-17.
10 Jackson PH, Stapleton JA, Russell MAH, Merriman RJ Predictors of outcome in a general practitioner intervention against smoking. Prev Med 1986; 15: 244-53.

11 Velicer WF, Prochaska JO, Rossi JS, Snow MG. Assessing outcome in smoking cessation studies. Psychol Bull 1992; 111: $23-41$.

12 Dean AG, Dean JA, Burton AH, Dicker RC. Epi info version $5-\mathrm{a}$ word processing, database and statistics version $5-$ a word processing, database and statistics system for epidemiology on microcompurer

13 Marlatt GA, Curry S, Gordon JR. A longitudinal analysis of unaided smoking cessation. $\mathcal{f}$ Consult Clin Psychol $1988 ; 56: 715-20$.

14 Gritz E, Carr CR, Marcus AC. Unaided smoking cessation: great American smokeout and new year's day quitters. Psychosoc Oncol 1988; 6: 217-34.

15 Smith FJ. Community Pharmacists and Health Promotion: a study of consultations between pharmacists and clients. Health Promotion Int 1992; $7: 249-55$.

16 Cartwright A, O'Brien $M$. Social class variations in health care in general practitioner consultations. In: Stacey $M$, ed. The sociology of the NHS. Sociological Review d. The sociog 2 Keele: University of Keele, 1976.

17 Bond CM, Taylor RJ, Sinclair HK, Winfield AJ. Some characteristics of Scottish community pharmacies: how characteristics of Scottish community pharmacies: how far has Nuffield

18 Kenford SL, Fiore MC, Jorenby DE, Smith SS, Wetter D, Baker TB. Predicting smoking cessation: who will quit with and without the nicotine patch. FAMA 1994; 271 : 589-94

19 Orleans CT et al. Use of transdermal nicotine in a statelevelprescription plan for the elderly: a first look at 'real world' patch users. FAMA 1994; 271: 601-7.

20 Hughes JR, Hatsukami DK, Skoog KP. Physical dependence on nicotine in gum. $f A M A 1986 ; 255$ : 3277-9.

21 Institute of Pharmacy Management International Conference Report : market research in community pharmacy. Pharm f 1991; 247: 612-3.

22 Royal Pharmaceutical Society. Guidelines on smoking cessation advice in the pharmacy. Pharm f 1994; 252: 816.

23 Pharmaceutical care: the future for community pharmacy. Report of the joint working party on the future role of the community pharmaceutical services. London: Royal Pharmaceutical Society on behalf of the Department of Health and the pharmaceutical profession, 1992.

24 British pharmacists to be paid for counselling, advice and referrals? [anonymous] Pharm f 1991; 247: 404.

25 Secretary of State for Health. The health of the nation: a strategy for health in England ( $\mathrm{Cm}$ 1986). London: strategy for

26 Lennox AS, Taylor RJ. Factors associated with outcome in unaided smoking cessation, and a comparison of those who have never tried to stop

27 Curry S, Thempson B, Sexton M, Omenn GS. Psychosocial predictors of outcome in a worksite smoking cessation programme. Am $\mathcal{F}$ Prev Med 1989; 14: 23-33. 